\title{
Non-Cooperative Spectrum Sensing Based on Cyclostationary Model of Digital Signals in the Context of Cognitive Radio
}

\author{
Jean-Marie Kadjo, Raoule Agoua, Aliou Bamba, Adama Konaté, Olivier Asseu \\ LASTIC, ESATIC, Abidjan, Côte d'Ivoire \\ Email: oasseu@yahoo.fr
}

How to cite this paper: Kadjo, J.-M., Agoua, R., Bamba, A., Konaté, A. and Asseu, O. (2021) Non-Cooperative Spectrum Sensing Based on Cyclostationary Model of Digital Signals in the Context of Cognitive Radio. Engineering, 13, 56-70. https://doi.org/10.4236/eng.2021.131005

Received: December 18, 2020

Accepted: January 24, 2021

Published: January 27, 2021

Copyright $\odot 2021$ by author(s) and Scientific Research Publishing Inc. This work is licensed under the Creative Commons Attribution International License (CC BY 4.0).

http://creativecommons.org/licenses/by/4.0/

\begin{abstract}
This paper addresses the problem of the opportunistic spectrum access in Cognitive Radio. Indeed, most spectrum sensing algorithms suffer from a high computational cost to achieve the detection process. They need a prior knowledge of signal characteristics and present a bad performance in low Signal to Noise Ratio (SNR) environment. The choice of the optimal detection threshold is another issue for these spectrum sensing algorithms. To overcome the limits of spectrum detectors, we propose in this paper, a blind detection method based on the cyclostationary features of communication signals. Our detector evaluates the level of hidden periodicity contained in the observed signal to make decision on the state of a bandwidth. In order to reduce the computational cost, we take advantage of the FFT Accumulation Method to estimate the cyclic spectrum of the observed signal. Then, we generate the Cyclic Domain Profile of the cyclic spectrum which allows us to evaluate the level of the hidden periodicity in the signal. This level of periodicity is quantified through the crest factor of Cyclic Domain Profile, which represents the decision statistic of the proposed detector. We have established the analytic expression of the optimal threshold of the detection and the probability of detection to evaluate the performance of the proposed detector. Simulation results show that the proposed detector is able to detect the presence of a communication signal on a bandwidth in a very low SNR scenario.
\end{abstract}

\section{Keywords}

Spectrum Sensing, Cyclostaionarity, FFT Accumulation Method, Cyclic Spectrum, Spectrum Coherence, Cognitive Radio

\section{Introduction}

In this paper, we propose the Cyclostationary Features based Detection (CFD) 
algorithm based on a spectral analysis of the cyclostationarity of digital communications signals. By definition, a cyclostationary process is a random process in which the statistical characteristics are periodic or quasi-periodic [1] [2] [3]. The strategy of detection based on cyclostationarity consists of developing algorithms to detect hidden periodicities of the observed signal. In digital communications, this cyclostationarity is due to coding, multiplexing and modulation techniques, which generate hidden frequencies in the spectrum of the transmitted signal [4] [5]. Since the detection of an unoccupied frequency band amounts to detect the presence or absence of a communication signal on this frequency band, a spectral analysis of the cyclostationarity of the received signal can then help in the detection of unoccupied spectral bands. The CFD acts in a non-cooperative context [6] [7]. It does not require a prior knowledge of the characteristics of the PU signal and remains effective in a very low SNR channel. Our cyclostationary detection approach is divided into two main stages:

- First, we adapt the Fast Fourier transform Accumulation Method (FAM) algorithm to estimate the cyclic spectrum of the observed signal.

- Then, we generate the Cyclic Domain Profile (CDP) which allows us to evaluate the level of the hidden periodicity in the signal. This level of periodicity is quantified through the Crest Factor (CF) of CDP, which represents the decision statistic of CFD.

During the detection phase, the CFD compares the CF of the received signal to a predetermined threshold. The simulations which we carried out through the Receiver Operating Characteristic (ROC) curves show the robustness of our detection method.

The rest of this paper is organized into five sections. In Section 2, we briefly present the properties of cyclostationary processes. Section 4 presents the FAM algorithm used to estimate the cyclic spectrum. The principle of the detector is presented in Section 5. We end this paper with Sections 6 and 7 which respectively present the results of our simulations and the conclusion.

\section{Cyclostationnarity}

The first works on the cyclostationarity of communication signals were published in 1958 by Bennett [5] [8]. Subsequently, Gudzenko [9], Markelov [10] and Gladyshev [11] will be interested in the theory of cyclostationarity and study various aspects including the non-parametric spectral analysis of processes Cyclostationnaire (CS) [9], the study of CS processes in a Gaussian context [10] and the analysis of processes Almost Cyclo-Stationnary (ACS) [11]. In a series of publications, Herbst provides tools for analyzing periodic or nearly periodic variance processes [12] [13] [14] [15]. Kampé de Férier [16] and Parzen [17] [18] for their part are interested in the spectral analysis of the cyclostationarity of natural phenomena and Monin [19] proposes the use of cyclostationarity in the analysis of weather data. One of the pioneers of cyclostationarity since the 1980s 
is William Gardner who, apart from the probabilistic theory of cyclostationarity developed by Gladyshev [20] [21] [22], lays the foundations of the non-probabilistic theory of cyclostationarity [1] [2] [3] [5] [23] [24]. This non-probabilistic theory consists of extracting sinusoidal components by a quadratic transformation of the cyclostationary signal. However, the two approaches (probabilistic and nonprobabilistic) give similar results and are linked by the cyclo-ergodicity of cyclostationary processes. This cyclo-ergodicity states that the limits of the temporal averages and the statistical averages of the cyclic characteristics converge towards the same values [25].

Today, cyclostationarity is exploited in various fields including telecommunications [5], mechanics [26] [27] and astronomy [28].

The cyclostationarity is defined as an extension of the concept of stationarity or as a special case of non-stationarity. Such a signal is defined by a periodicity at order $\mathrm{n}$ of its statistics [29]. A CS or ACS process is defined in the strict sense or in the broad sense.

Let $X(t)$ be a real-value continuous-time stochastic process. The process $X(t)$ is said to be Cyclo-Stationary (CS) with period $T_{0}$ in the strict sense of the order $n$ if and only if its probabilistic characteristics are $T_{0}$-periodic [5]. Which means in other words that: $\forall t \in \mathbb{R}, \forall k \in \mathbb{Z}, \forall x \in \mathbb{R}^{n}, \exists T_{0} \in \mathbb{R}^{+}$:

$$
F_{X(t)}(x)=F_{X\left(t+k T_{0}\right)}(x)
$$

When the process $X(t)$ contains several periodicities, the process is named a polycyclostationary process. Thus, $X(t)$ is said to be polyclostationary in the strict sense with the periods $T_{0}, T_{1}, \cdots, T_{P}$ if and only if its statistical properties are periodic in time with the periods $T_{0}, T_{1}, \cdots, T_{P}$ [5] [25].

Let $X(t)$ be a real-value continuous-time stochastic process. The process $X(t)$ is said to be CS of period $T_{0}$ in the broad sense if and only if its statistics of order 1 (its mean) and of order 2 (its autocorrelation function) vary periodically over time with a period $T_{0}$.

In the rest of this paper, we will focus on cyclostationarity in the wide sense to develop our CFD detector which only exploits the statistical properties of second order of the digital communications signal.

The theory of second order cyclostationarity is based on the autocorrelation function of the stochastic process. Considering that in practice, the observed signal is only one realization of the cyclostationary process, we will deal in the continuation with the second order cyclostationarity of digital communications signals.

A centered signal $x(t)$ is said to be second-order cyclostationary, in the wide sense, if its autocorrelation function $R_{x}(t, \tau)$ defined by:

$$
R_{x}(t, \tau) \triangleq E\left[x\left(t+\frac{\tau}{2}\right) x^{*}\left(t-\frac{\tau}{2}\right)\right]
$$

is a $T_{0}$-periodic function for each $\tau$; which means in other words that: $\forall k \in \mathbb{Z}^{*}$, 


$$
R_{x}\left(t+k T_{0}, \tau\right)=R_{x}(t, \tau)
$$

where $\tau$ denotes the time offset (correlation variable). $T_{0}$ is called the cyclic period. It is a period hidden in the signal $x(t) ; E[$.$] denotes the expectation$ [30].

Since the auto-correlation function $R_{x}(t, \tau)$ is $T_{0}$-periodic in $t$ for each shift $\tau$, then it can therefore be decomposed into Fourier series in the form:

$$
R_{x}(t, \tau)=\sum_{k=1}^{K} R_{x}^{k \alpha_{0}}(\tau) \cdot \mathrm{e}^{j 2 \pi k \alpha_{0} t}
$$

where $\alpha_{0}=1 / T_{0}$ denotes the fundamental cyclic frequency and $K$ the number of harmonics.

The quantity $R_{x}^{k \alpha_{0}}(\tau)$ is called the cyclic correlation function [24]. According to [24], $R_{x}^{k \alpha_{0}}(\tau)$ is given by:

$$
R_{x}^{k \alpha_{0}}(\tau) \triangleq \frac{1}{T_{0}} \int_{-\frac{T_{0}}{2}}^{\frac{T_{0}}{2}} R_{\chi}(t, \tau) \cdot \mathrm{e}^{-j 2 \pi k \alpha_{0} t} \mathrm{~d} t
$$

When its autocorrelation function contains several cyclic periods (hidden periodicities) $T_{0}, T_{1}, T_{2}, \cdots$, the signal $x(t)$ is said to be polycyclostationary [5] [31]. The polycyclostationary model is most suitable for digital communications signals. Thus, by adopting the polycyclostationary model for the signal $x(t)$, the autocorrelation function $R_{x}(t, \tau)$ of $x(t)$ becomes [31] [32]:

$$
R_{x}(t, \tau) \triangleq \sum_{\alpha \in \mathcal{A}} R_{x}^{\alpha}(\tau) \cdot \mathrm{e}^{j 2 \pi \alpha t}
$$

where $\mathcal{A}=\left\{\alpha_{0}, \alpha_{1}, \cdots, \alpha_{N}\right\}$ is a numbered set containing all the possible cyclic frequencies $\alpha$ and the Fourier coefficients $R_{x}^{\alpha}(\tau)$.

$$
R_{x}^{\alpha}(\tau) \triangleq \lim _{T \rightarrow \infty} \frac{1}{T} \int_{-\frac{T}{2}}^{\frac{T}{2}} R_{x}(t, \tau) \cdot \mathrm{e}^{-j 2 \pi \alpha t} \mathrm{~d} t
$$

denote the cyclic autocorrelation function.

By analogy with the Power Spectral Density, the Fourier transform of the cyclic autocorrelation function is called density function of the cyclic correlation or cyclic spectrum [24]:

$$
S_{x}^{\alpha}(v) \triangleq \int_{-\infty}^{+\infty} R_{x}^{\alpha}(\tau) \cdot \mathrm{e}^{-j 2 \pi v \tau} \mathrm{d} \tau
$$

The estimation of the quantities defined by Equations (7) and (8) constitutes the cyclic spectral analysis.

\section{Cyclic Spectral Analysis}

Let $w(t)$ be a sliding window of unit area and not zero over the interval $\left[\frac{-T}{2}, \frac{T}{2}\right]$. The adequate estimator of the cyclic autocorrelation function $R_{x}^{\alpha}(\tau)$ of the observation $x(t)$ for $t \in\left[t_{0}-T / 2, t_{0}+T / 2\right]$ is the cyclic correlogram $R_{x}^{(T)}(\alpha, t)$ defined by [31]:

$$
R_{x}^{(T)}(\alpha, \tau) \triangleq \int_{\mathbb{R}} w\left(t-t_{0}\right) x(t+\tau) \bar{x}(t) \mathrm{e}^{-j 2 \pi \alpha t} \mathrm{~d} t
$$


$\bar{x}$ represents the conjugate of $x(t)$. The cyclic correlogram $R_{x}^{(T)}(\alpha, \tau)$ is a consistent estimator of $R_{x}^{\alpha}(\tau)$ [31]. The letter $T$ superscript of $R_{x}^{(T)}(\alpha, \tau)$ denotes the width of the sliding window $w(t)$.

Assuming $w(t)=\operatorname{rect}(t / T) / T$, we show that the cyclic autocorrelation function is equivalent to [3] [33]:

$$
R_{x}^{(T)}(\alpha, \tau)=\frac{1}{T} \int_{-\frac{T}{2}}^{+\frac{T}{2}} x\left(t+\frac{\tau}{2}\right) \bar{x}\left(t-\frac{\tau}{2}\right) \cdot \mathrm{e}^{-j 2 \pi \alpha t} \mathrm{~d} t
$$

By introducing $\mathrm{e}^{-j \pi \alpha\left(\frac{\tau}{2}-\frac{\tau}{2}\right)}=1$ into the equation eqref Equation Autocor Cyclic2, we end up with:

$$
\begin{aligned}
R_{x}^{(T)}(\alpha, \tau) & =\frac{1}{T} \int_{-\frac{T}{2}}^{\frac{T}{2}} x\left(t+\frac{\tau}{2}\right) \mathrm{e}^{-j 2 \pi \alpha\left(t+\frac{\tau}{2}\right)} \bar{x}\left(t-\frac{\tau}{2}\right) \mathrm{e}^{-j 2 \pi \alpha\left(t-\frac{\tau}{2}\right)} \mathrm{d} t \\
& =\frac{1}{T}\{z(\alpha, \tau) * \bar{z}(\alpha,-\tau)\}
\end{aligned}
$$

where $z(\alpha, \tau)=x\left(t+\frac{\tau}{2}\right) \mathrm{e}^{-j 2 \pi \alpha\left(t+\frac{\tau}{2}\right)}$ and ${ }^{\star}$ denotes the convolution product operator.

The Fourier transform of the cyclic correlogram $R_{x}^{(T)}(\alpha, \tau)$ gives the cyclic periodogram $I^{(T)}(\alpha, v)$ [31]:

$$
\begin{aligned}
I^{(T)}(\alpha, v ; t) & \triangleq \frac{1}{T} T F\left\{R_{x}^{(T)}(\alpha, \tau)\right\} \\
& =\frac{1}{T} T F\{z(\alpha, \tau) * \bar{z}(\alpha,-\tau)\} \\
& =\frac{1}{T} X_{T}\left(t, v+\frac{\alpha}{2}\right) \bar{X}_{T}\left(t, v-\frac{\alpha}{2}\right)
\end{aligned}
$$

where $X_{T}(t, v)$ is the Short Term Fourier Transform of $x(t)$ defined as follows:

$$
X_{T}(t, v) \triangleq \int_{t-\frac{T}{2}}^{t+\frac{T}{2}} x(u) \mathrm{e}^{-j 2 \pi v u} \mathrm{~d} u
$$

$X_{T}\left(t, v+\frac{\alpha}{2}\right)$ is named the complex demodulate [5] [34].

The cyclic periodogram $I^{(T)}(\alpha, v ; t)$ is a non-consistent estimator of the cyclic spectrum $S_{x}^{\alpha}(v)$. As in the case of stationary processes, consistency can be obtained by temporal or frequency averaging of $I^{(T)}(\alpha, v ; t)$. We then obtain two estimators of the cyclic spectrum: the frequency-averaged cyclic periodogram $G_{x}^{(\Delta t, \Delta v)}(\alpha, v)$ and the time-averaged cyclic periodogram $S_{x}^{(\Delta t, \Delta v)}(\alpha, v)$ [31]:

$$
\begin{aligned}
G_{x}^{(\Delta t, \Delta v)}(\alpha, v) & =\frac{1}{\Delta v} \int_{v-\Delta v / 2}^{v+\Delta v / 2} I^{(\Delta t)}(\alpha, \lambda ; t) \mathrm{d} \lambda \\
S_{x}^{(\Delta t, \Delta v)}(\alpha, v) & =\frac{1}{\Delta t} \int_{t_{0}-\Delta t / 2}^{t_{0}+\Delta t / 2} I^{(1 / \Delta v)}(\alpha, v ; t) \mathrm{d} t
\end{aligned}
$$

with $\Delta t$ the duration of observation of the signal $x(t)$ and $\Delta v$ the frequency resolution.

$S_{x}^{(\Delta t, \Delta v)}(\alpha, v)$ and $G_{x}^{(\Delta t, \Delta v)}(\alpha, v)$ are defined by a frequency resolution $\Delta v$ 
and a cyclic frequency resolution $\Delta \alpha$.

$\Delta t$ denotes the duration of observation of the signal $x(t)$ and $T$ the duration (or width) of the sliding window (apodization window), we have [5] [23] [31]:

$$
\begin{gathered}
\Delta \alpha=\frac{1}{\Delta t} \\
\Delta v=\frac{1}{T}
\end{gathered}
$$

To obtain a fine estimate of the cyclic spectrum, the Grenander uncertainty condition must be respected [35]:

$$
\Delta t \cdot \Delta v \gg 1
$$

It states that for a better estimate of the cyclic spectrum, the duration $T$ of the apodization window $w(t)$ must be much less than the observation duration $\Delta t$ of the signal $x(t)$ to analyze [35].

The estimators $S_{x}^{(\Delta t, \Delta v)}(\alpha, v)$ and $G_{x}^{(\Delta t, \Delta v)}(\alpha, v)$ of $S_{x}^{\alpha}(v)$ are asymptotically equivalent [31]:

$$
\lim _{\Delta v \rightarrow 0} \lim _{\Delta t \rightarrow \infty} S_{x}^{(\Delta t, \Delta v)}(\alpha, v)=\lim _{\Delta v \rightarrow 0} \lim _{\Delta t \rightarrow \infty} G_{x}^{(\Delta t, \Delta v)}(\alpha, v)=S_{x}^{\alpha}(v)
$$

However, in [34] [35] [36], the authors show that the cyclic spectrum estimators based on temporal smoothing are more efficient in terms of computation time and provide a finer estimate.. Consequently, we will focus in the rest of our work in methods based on temporal smoothing. The most used temporal smoothing algorithms are:

- FAM [36] [37] algorithm;

- Spectral Correlation Algorithm (SSCA) algorithm [36] [37].

The FAM algorithm is faster and more efficient than the SSCA algorithm. Our detection model therefore uses the FAM algorithm to estimate the cyclic spectrum of the observed signal.

\section{FFT Accumulation Method}

Let $x(n), n \in \mathbb{Z}$ be the discrete signal obtained by uniform sampling of the continuous signal $x(t), t \in \mathbb{R}$. Let $N$ be the number of samples observed during the duration $\Delta t, \Delta t=N T_{e}$ and $N_{P}$ the number of samples contained in the sliding window $w(n) ; T=N_{P} T_{e} ; T_{e}$ denotes the sampling period. The cyclic spectrum estimator by time averaging is written [5] [6] [38]:

$$
\begin{aligned}
S_{x}^{\left(N, \frac{1}{N_{P}}\right)}(\alpha, v)= & \frac{1}{N} \sum_{n=0}^{N-1} \frac{1}{N_{P}} X_{T}\left(n, v+\frac{\alpha}{2}\right) \bar{X}\left(n, v-\frac{\alpha}{2}\right) \\
= & \frac{1}{N} \sum_{n=0}^{N-1} \frac{1}{N_{P}} T F\left\{w(k) x(n-k) \mathrm{e}^{-j 2 \pi\left(\frac{\alpha}{2}\right)(n-k) T_{e}}\right\} \\
& \operatorname{TF}\left\{[\overline{w(k) x(n-k)}] \mathrm{e}^{+j 2 \pi\left(\frac{\alpha}{2}\right)(n-k) T_{e}}\right\}
\end{aligned}
$$


The FAM algorithm for Fast Fourier Transform Accumulation Method, based on Equation (20), takes advantage of the advantages of the Fast Fourier Transform (Fast Forier Transform (FFT)) to reduce the computational cost of the spectrum cyclic. It divides the dual-frequency plane $(v, \alpha)$ into sub-blocks $\left(v_{i}, \alpha_{i}\right)$, then calculates the estimates of the cyclic spectrum $S_{x}^{\left(N, \frac{1}{N_{P}}\right)}\left(\alpha_{i}, v_{i}\right)$ of each sub-block using the FFT. It is constitued of three main stages:

1) The computation of complex demodulates $X_{T}\left(n, v+\frac{\alpha}{2}\right)$ and of their conjugates $\bar{X}_{T}\left(n, v-\frac{\alpha}{2}\right)$ to obtain the spectral composition of the signal.

2) The two-by-two product between complex demodules and their conjugates corresponds to the calculation of the cyclic correlogram

$$
I^{(T)}(\alpha, v ; n)=\frac{1}{T} X_{T}\left(n, v+\frac{\alpha}{2}\right) \bar{X}_{T}\left(n, v-\frac{\alpha}{2}\right) .
$$

3) The computation of FFT of $I^{(T)}(\alpha, v ; n)$ to obtain the estimation of cyclic spectrum $S_{x}^{\left(N, \frac{1}{N_{P}}\right)}(\alpha, v)$.

Figure 1 summerizes the different stages of FAM algorithm.

\section{Detection Modeling}

Let $s(t)$ be the signal of the primary user (PU) and $y(t)$ the signal observed by the secondary user (SU):

$$
y(t)=\theta \cdot h(t) s(t)+b(t)
$$

where $h(t)$ denotes the impulse response of the channel, $\theta$ a Boolean variable and $b(t)$ a Gaussian white noise of variance $\sigma_{b}^{2}$.

The hypotheses $\mathcal{H}_{0}$ and $\mathcal{H}_{1}$ are as follows:

- $\mathcal{H}_{0}$ : no communication signal; $\theta=0$;

- $\mathcal{H}_{1}$ : communication signal presence; $\theta=1$.

The fundamental idea of detection consists in measuring the level of hidden periodicity in the signal $y(t)$.

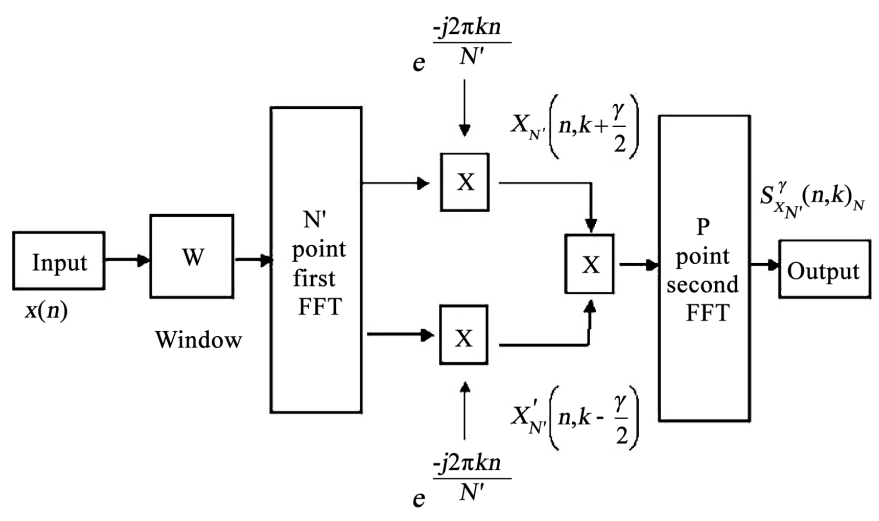

Figure 1. Synotic diagram of the FAM algorithm inspired by [35]. 
To measure this hidden periodicity, we first define the Cyclic Domain Profile or CDP $D(\alpha)$ which contains the maximum values of the cyclic spectrum $S_{y}^{\alpha}(v)$ :

$$
D(\alpha)=\max _{v}\left\|S_{y}^{\alpha}(v)\right\|
$$

Next, we define the crest factor $F_{c}$ of the CDP $D(\alpha)$ which will serve as the decision statistic $\mathcal{T}_{\text {CFD }}$ of the CFD:

$$
\mathcal{T}_{\text {CFD }}=F_{c}=\frac{\max _{\alpha} D(\alpha)}{\sqrt{\frac{1}{2 N} \sum_{k=1}^{2 N} D^{2}\left(\alpha_{k}\right)}}
$$

The detection criterion is given by:

$$
\mathcal{T}_{\text {CFD }} \stackrel{\mathcal{H}_{1}}{\gtrless} \lambda_{\mathcal{H}_{0}} \lambda_{\text {CFD }}
$$

$\lambda_{C F D}$ is the detection threshold predetermined according to the desired $P_{f a}$ [39].

In order to analytically approach the optimal detection threshold $\lambda_{C F D}$, we must estimate the Probability Density Function (PDF) of the crest factor $F_{c}$ under $\mathcal{H}_{0}$. Equation (23) shows that the calculation of $F_{c}$ is based on a maximization function; we can therefore approach the PDF of $F_{c}$ both under $\mathcal{H}_{0}$ and under $\mathcal{H}_{1}$ by the PDF $f_{Z}(z)$ of generalized extreme value or Generalized Extreme Value (GEV) of parameters ( $\mu, \sigma, \xi)$ defined by [40] [41] [42]:

$$
f_{Z}(z)=\frac{1}{\sigma}\left(1+\xi \frac{z-\mu}{\sigma}\right)^{\left(-\frac{1}{\xi}-1\right)} \exp \left[-\left(1+\xi \frac{z-\mu}{\sigma}\right)^{-1 / \xi}\right]
$$

with $\mu$ location parameter; $\sigma$ the scale parameter and $\xi \in \mathbb{R}$ the shape parameter.

Figure 2 shows us the evolution of $F_{c}$ under $\mathcal{H}_{0}$ and under $\mathcal{H}_{1}$ for a SNR $=-11 \mathrm{~dB}$. We define the probability of false alarm $P_{f a}$ by:

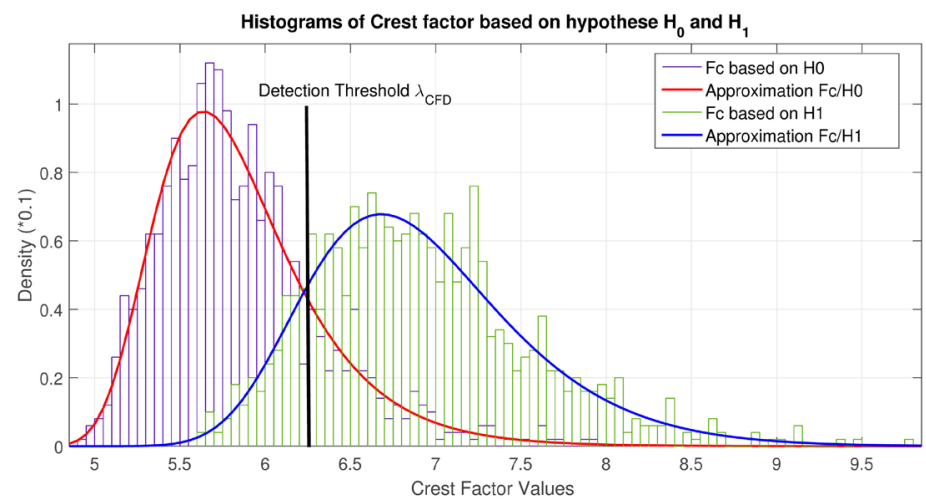

Figure 2. Approximation of the distribution of $F_{c}$ under $\mathcal{H}_{0}$ and under $\mathcal{H}_{1}$. Under $H_{1}$, the signal to noise ratio is SNR $=-11 \mathrm{~dB} . F_{c}$ can be approximated by the PDF of generalized extreme values (GEV for Generalized Extreme Value). Depending on the probability of false alarm for the detector, we can set the detection threshold $F_{c_{0}}$. 


$$
P_{f a}=\int_{\lambda_{C F D}}^{+\infty} f_{Z / H_{0}}(z) \mathrm{d} z
$$

with:

$$
\left\{\begin{array}{l}
\mu_{0}=\mu / \mathcal{H}_{0} \\
\sigma_{0}=\sigma / \mathcal{H}_{0} \\
\xi_{0}=\xi / \mathcal{H}_{0}
\end{array}\right.
$$

$P_{f a}$ becomes as follows:

$$
P_{f a}=\int_{\lambda_{C F D}}^{+\infty} \frac{1}{\sigma_{0}}\left[1+\xi_{0} \frac{z-\mu_{0}}{\sigma_{0}}\right]^{-\frac{1}{\xi_{0}}-1} \exp \left[-\left(1+\xi_{0} \frac{z-\mu_{0}}{\sigma_{0}}\right)^{-1 / \xi_{0}}\right] \mathrm{d} z
$$

Using the change of variable $u=1+\xi_{0} \frac{z-\mu_{0}}{\sigma_{0}}$, we obtain:

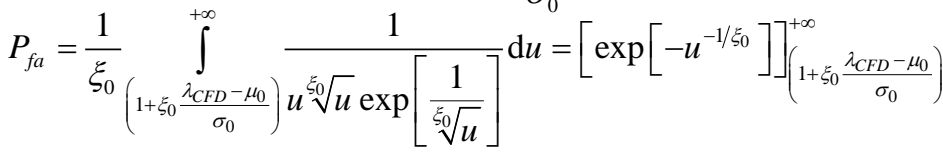

$$
\begin{aligned}
& P_{f a}=1-\exp \left[-\left(1+\xi_{0} \frac{\lambda_{C F D}-\mu_{0}}{\sigma_{0}}\right)^{-1 / \xi_{0}}\right]
\end{aligned}
$$

From Equation (29), we determine the optimal detection threshold $\lambda_{C F D}$ as follows:

$$
\lambda_{C F D}=\frac{\sigma_{0}}{\xi_{0}}\left[\left(\ln \left(\frac{1}{1-P_{f a}}\right)\right)^{-\xi_{0}}-1\right]+\mu_{0}
$$

With:

$$
\left\{\begin{array}{l}
\mu_{1}=\mu / H_{1} \\
\sigma_{1}=\sigma / H_{1} \\
\xi_{1}=\xi / H_{1}
\end{array}\right.
$$

the probability of detection $P_{d}$ can be defined by:

$$
\begin{aligned}
& P_{d}=\int_{\lambda}^{+\infty} f_{Z / H_{1}}(z) \mathrm{d} z \\
& P_{d}=1-\mathrm{e}^{-\left[1+\xi_{1} \frac{\lambda-\mu_{1}}{\sigma_{1}}\right]^{-1 / \xi_{1}}}
\end{aligned}
$$

Equations (29) and (32) allow to establish the relation between $P_{d}$ et al. $P_{f a}$ :

$$
P_{d}=1-\exp \left\{-\left[1+\xi_{1} \frac{\frac{\sigma_{0}}{\xi_{0}}\left[\left(\ln \left(\frac{1}{1-P_{f a}}\right)\right)^{-\xi_{0}}-1\right]+\mu_{0}-\mu_{1}}{\sigma_{1}}\right]^{-1 / \xi_{1}}\right\}
$$

A delicate part in this approximation is the estimation of the parameters $\xi, \sigma, k$ of the probability density function GEV. To do this, we use the Optimal Biased Robust Estimator (OBRE) algorithm proposed in [40] to estimate the parameters $\mu, \sigma$ and $\xi$. OBRE is a robust estimator based on Maximum Like- 
lihood or gls mle. Table 1 gives some values of the parameters $\mu, \sigma$ and $\xi$ of the GEV probability density function of $F_{c}$ under $\mathcal{H}_{1}$ obtained for different values of SNR.

\section{Simulations and Results}

In order to compare the theoretical and experimental results, we generate the performance curves $P_{d}$ as a function of Signal Noise Ratio (SNR) and the Reciever Operating Characteristics (ROC) curves. These simulations are performed on different types of signals (16-QAM, QPSK, BPSK, 4-ASK). We group the simulation parameters in Table 2.

Figure 3 shows a concordance between the theoretical and experimental performance curves $P_{d}$ as a function of SNR for a 16-QAM signal. We generated the theoretical curve from Equation (33) and the experimental curve using Monte Carlo simulations (1000 iterations). Thus according to the theory, our detector is able to detect a communication signal in a Gaussian channel as soon as the signal to noise ratio SNR $\geq-11 \mathrm{~dB}$ with a detection probability $P_{d}=0.9$ for a $P_{f a}=0.05$. These results are consistent with those obtained experimentally.

To analyse the evolution of the $P_{d}$ as a function of the $P_{f a}$, we use the roc curves. Figure 4 gives the theoretical and experimental ROC curves of the CFD on a 16-QAM signal in a Gaussian channel with a SNR $=-11 \mathrm{~dB}$. The theoretical curve is generated by Equation (33) and the experimental curve is obtained by Monte Carlo simulations. We can note through these results the similarity between the two curves.

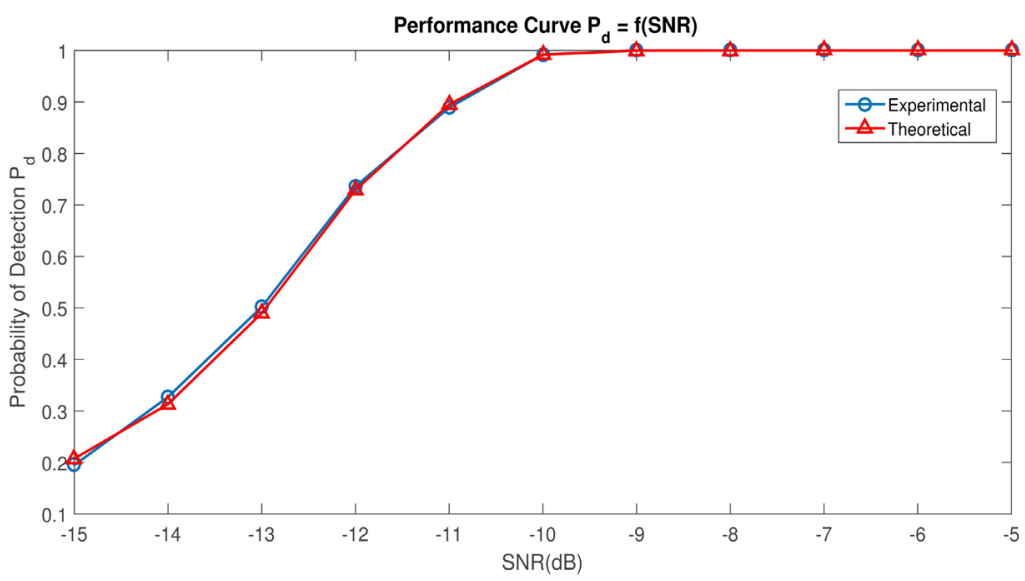

Figure 3. $P_{d}$ performance curve as a function of $S N R$. The theoretical curve obtained according to Equation (33) and the experimental one coincide.

Table 1. Value of $\mu, \sigma$ and $\xi$ under $H_{1}$ based on the SNR.

\begin{tabular}{cccccccc}
\hline $\mathrm{SNR}(\mathrm{dB})$ & -14 & -12 & -10 & -9 & -8 & -7 & -6 \\
\hline$\xi_{1}$ & -0.033 & -0.104 & -0.098 & -0.124 & -0.1016 & -0.0804 & -0.1286 \\
$\sigma_{1}$ & 0.439 & 0.528 & 0.557 & 0.627 & 0.647 & 0.663 & 0.718 \\
$\mu_{1}$ & 5.76 & 6.326 & 7.126 & 7.673 & 8.19 & 8.839 & 9.533 \\
\hline
\end{tabular}


The detector based on the cyclostationary characteristics of the signal acts in a quasi-blind context. It has no knowledge of the characteristics of the signal emitted by the PU. Therefore, we compare it to the classic detector based on the energy level. We notice that the probability of detection of the CFD is almost identical to that of the Energy-based Detection (ED). For a false alarm probability set at $P_{f a}=0.05$, we obtained (Figure 5) $P_{d_{C F D}}=0.99$ and $P_{d_{E D}}=0.97$ in a channel where the $\mathrm{SNR}=-10 \mathrm{~dB}$.

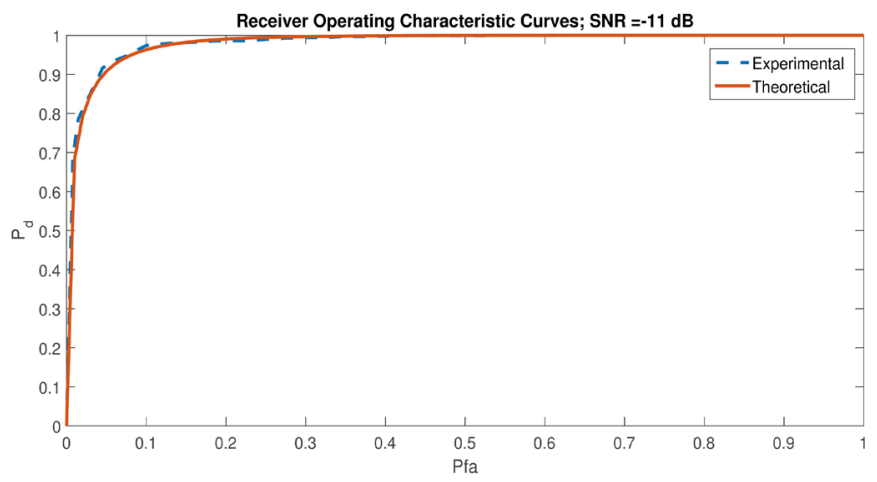

Figure 4. Receiver Operating Characteristic (ROC) of Cyclostationary Features based Detector (CFD) in Gaussian channel with SNR $=-11 \mathrm{~dB}$ for 16-QAM signal.

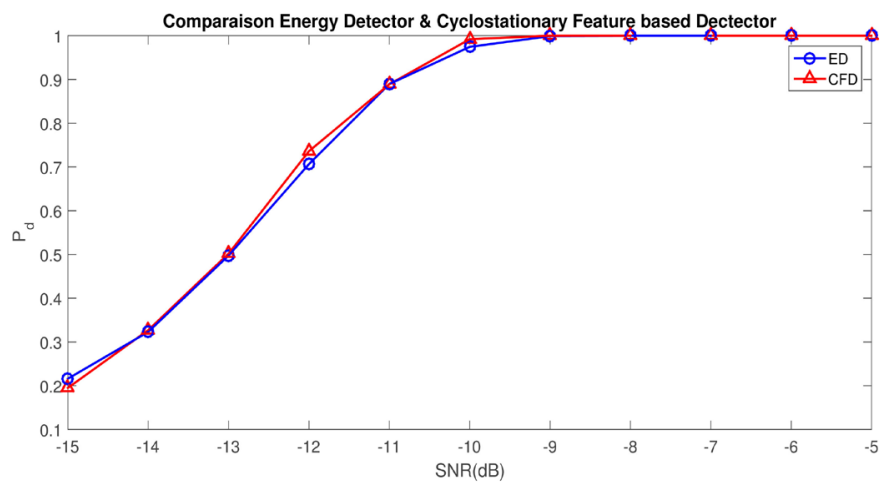

Figure 5. Comparison of cyclostationary feature based detector and energy detector. For $P_{f a}=0.05$, we obtain $P_{d_{C F D}}=0.99$ and $P_{d_{E D}}=0.97$ in a channel where $S N R=-10 \mathrm{~dB}$.

Table 2. Simulation parameters of CFD.

\begin{tabular}{ccc}
\hline & 64-QAM, 16-QAM, BPSK, 4-ASK Signal & \\
\hline User & Parameters & Values \\
\hline \multirow{2}{*}{ Primary } & Sampling rate $F_{e}$ & $128 \mathrm{kHz}$ \\
& Data rate & $16 \mathrm{kHz}$ \\
& bandwidth $W$ & $21.6 \mathrm{kHz}$ \\
\hline \multirow{2}{*}{ Secondary } & Observation duration $\Delta t$ & $16 \mathrm{~ms}$ \\
& Number of samples $N$ & 2048 \\
\hline \multirow{2}{*}{ Frequency resolution } & $\Delta \alpha$ & $62.5 \mathrm{~Hz}$ \\
& $\Delta v$ & $1 \mathrm{kHz}$ \\
\hline
\end{tabular}




\section{Conclusion}

Detecting the Primary User (PU) signal, in a very weak SNR condition, is one of the most difficult tasks in cognitive radio. In this paper, we have proposed a non-cooperative detection model which is able to detect the signal of PU in low SNR scenario. This detection model exploits the cyclostationary features of communication signals. This cyclostationarity can be revealed through cyclic spectral analysis. Thus, after the estimation of the cyclic spectrum using the FFT Accumulation Method (FAM) algorithm, we generate the Cyclic Domain Profile (CDP). Then, we quantify this level of periodicity using the CDP crest factor. We use the CDP crest factor as the decision statistic to detect the state of the channel (busy or not). We approached the probability density function of the crest factor, which allowed us to analytically determine the optimal detection threshold, the probabilities of false alarm and detection. The simulations carried out subsequently show a strong similarity between the theoretical results and the experimental results. Cyclostationary Feature based Detection is an efficient detector. In low SNR scenario, it is able to detect a digital communication signal up to $\mathrm{SNR}=-11 \mathrm{~dB}$. Our work on CFD shows that the CDP makes it possible to visualize the hidden frequencies of the observed signal on the one hand and on the other hand that the CF is a characteristic quantity of the modulation technique. We propose, in future works, to exploit these two tools (the CDP and the Crest Factor) to develop an algorithm, which is able to detect the signal of the PU and also to distinguish the signal of a PU from the signal of a SU, by using the principle of modulation recognition.

\section{Conflicts of Interest}

The authors declare no conflicts of interest regarding the publication of this paper.

\section{References}

[1] Gardner, W. (1986) Introduction to Random Process with Applications to Signals and Systems. Gardner, W., Ed., North-Holland, Macmillan, New York.

[2] Gardner, W.A. (1986) Statistical Spectral Analysis: A Nonprobabilistic Theory. Prentice-Hall, Inc., Tokyo.

[3] Gardner, W. (1991) Exploitation of Spectral Redundancy in Cyclostationary Signals. IEEE Signal Processing Magazine, 8, 14-36. https://doi.org/10.1109/79.81007

[4] Prithiviraj, V., Sarankumar, B., Kalaiyarasan, A., Praveen, P. and Signh, N. (2011) Cyclostionary Analysis Method of Spectrum Sensing for Cognitive Radio. Wireless VITAE, IEEE, Chennai, India, 1-5. https://doi.org/10.1109/WIRELESSVITAE.2011.5940821

[5] Gardner, W. (1994) Cyclostationarty in Communications and Signal Processing. IEEE Press, New York.

[6] Tom, C. (1995) Investigation and implementation of computationally-efficient algorithm for cyclic spectral analysis. Master's Thesis, Carleton University Ottawa, Ontario, Canada. 
[7] Jang, M. (2014) Blind Cyclostationary Spectrum Sensing in Cognitive Radios. IEEE Communications Letters, 8, 393-396. https://doi.org/10.1109/LCOMM.2014.012714.132507

[8] Bennett, W.R. (1958) Statistics of Regenerative Digital Transmission. The Bell System Technical Journal, 37, 1501-1542. https://doi.org/10.1002/j.1538-7305.1958.tb01560.x

[9] Gudzenko, L. (1959) On Periodically Nonstationary Processes. Radiotekhnika i Electronika, 4, 1062-1064.

[10] Markelov, V. (1966) Axis Crossings and Relative Time of Existence of a Periodically Nonstationary Random Process. Soviet Radiophysics, 9, 440-443. https://doi.org/10.1007/BF01046009

[11] Gladyshev, E. (1961) Periodically Correlated Random Sequence. Soviet Mathematics, 2, 385-388.

[12] Herbst, L., et al. (1963) Almost Periodic Variances. The Annals of Mathematical Statistics, 34, 1549-1557. https://doi.org/10.1214/aoms/1177703887

[13] Herbst, L.J. (1963) Periodogram Analysis and Variance Uctuations. Journal of the Royal Statistical Society. Series B (Methodological), 25, 442-450. https://doi.org/10.1111/j.2517-6161.1963.tb00527.x

[14] Herbst, L. (1965) The Statistical Fourier Analysis of Variances. Journal of the Royal Statistical Society: Series B (Methodological), 27, 159-165.

https://doi.org/10.1111/j.2517-6161.1965.tb00598.x

[15] Herbst, L.J. (1964) Spectral Analysis in the Presence of Variance Uctuations. Journal of the Royal Statistical Society. Series B (Methodological), 26, 354-360. https://doi.org/10.1111/j.2517-6161.1964.tb00568.x

[16] de Feriet, J.K. (1962) Correlation and Spectrum of Asymptotically Stationary Random Functions. Mathematical Studies, 30, 55-67.

[17] Parzen, E. (1963) On Spectral Analysis with Missing Observations and Amplitude Modulation. Sankhyā: The Indian Journal of Statistics, Series A, 25, 383-392.

[18] (1961) Spectral Analysis of asymptotically Stationary Time Series. Stanford University Calif. Applied Mathematics and Statistics Lab, Tech. Rep.

[19] Monin, A. (1963) Stationary and Periodic Time Series in the General Circulation of the Atmosphere. Proceedings of the Symposium on Time Series Analysis, Wiley, New York, 144-151.

[20] Gladyshev, E. (1963) Periodically and Almost-Periodically Correlated Random Processes with a Continuous Time Parameter. Theory of Probability Its Applications, 8, 173-177. https://doi.org/10.1137/1108016

[21] Dehay, D. and Hurd, H. (1994) Representation and Estimation for Periodically and Almost Periodically Correlated Random Processes. Cyclostationarity in Communications and Signal Processing, IEEE Press, New York, 295-328.

[22] Dandawate, A.V. and Giannakis, G.B. (1995) Asymptotic Theory of Mixed Time Averages and kth-Order Cyclic-Moment and Cumulant Statistics. IEEE Transactions on Information Theory, 41, 216-232.

https://doi.org/10.1109/18.370106

[23] Gardner, W. (1986) The Spectral Correlation Theory of Cyclostationary Time-Series. Signal Processing, Elsevier Science Publishers, 11, 13-36. https://doi.org/10.1016/0165-1684(86)90092-7

[24] Gardner, W. and Spooner, C. (1992) Signal Interception: Performance Advantages of Cyclic-Feature Detector. IEEE Transactions on Communications, 40, 149-159. 
https://doi.org/10.1109/26.126716

[25] Rostaing, P. (1997) Détection de signaux modulés en exploitant leurs propriétés cyclostationnaires: Application aux signaux sonar. Ph.D. Dissertation, Université de Nice, Nice.

[26] Capdessus, C. (1992) Aide au diagnostic des machines tournantes par traitement du signal. Ph.D. Dissertation, Institut National Polytechnique de Grenoble, Grenoble.

[27] Randall, R.B., Antoni, J. and Chobsaard, S. (2001) The Relationship between Spectral Correlation and Envelope Analysis in the Diagnostics of Bearing Faults and Other Cyclostationary Machine Signals. Mechanical Systems and Signal Processing, 15, 945-962. https://doi.org/10.1006/mssp.2001.1415

[28] Weber, R. and Faye, C. (1998) Real Time Detector for Cyclostationary Rfi in Radio Astronomy. 9th European Signal Processing Conference (EUSIPCO 1998), Island of Rhodes, 8-11 September 1998, 1-4.

[29] Roussel, J. (2014) Modélisation cyclostationnaire et séparation de sources des signaux électromyographiques. Ph.D. dissertation, Université d'Orléans, France.

[30] Sutton, P.D., Nolan, K.E. and Doyles, L.E. (2008) Cyclostationary Signatures in Pratical Cognitive Radio Applications. IEEE Journal on Selected Areas in Communications, 26, 13-24. https://doi.org/10.1109/JSAC.2008.080103

[31] Napolitano, A. (2016) Cyclostationarity: New Trends and Applications. Signal Processing, 120, 385-408. https://doi.org/10.1016/j.sigpro.2015.09.011

[32] Kadjo, J.-M., Yao, K.C. and Mansour, A. (2016) Blind Detection of Cyclostationary Features in the Context of Cognitive Radio. IEEE International Symposium on Signal Processing and Information Technology (ISSPIT), Limassol, Cyprus, December 2016, 150-155. https://doi.org/10.1109/ISSPIT.2016.7886025

[33] Brown, W. (1987) On the Theory of Cyclostationary Signals. Ph.D. Dissertation, University of California, Davis, California, USA.

[34] Brown, W. and Loomis, H. (1993) Digital Implementation of Spectral Correlation Analyzers. IEEE Transactions on Signal Processing, 41, 703-720.

https://doi.org/10.1109/78.193211

[35] Pace, P. (2009) Detecting and Classifying Low Probability of Intercept Radar. 2nd Edition, House, A., Ed., Artech House, Boston, London.

[36] Robert, R., Brown, W. and Loomis, H. (1991) Computationally Efficient Algorithms for Cyclic Spectral Analysis. Signal Processing Magazine, 8, 38-49.

https://doi.org/10.1109/79.81008

[37] (1993) A Review of Digital Spectral Correlation Analysis: Theory and Implementation. Article 6 in Part II of Cyclostationarity in Communications and Signal Processing, Gardner, W.A., Ed., IEEE Press, New York, 455-479.

[38] Tom, C. (1995) Cyclostationary Spectral Analysis of Typical Satcom Signals Using the FFT Accumulation Method. Defence Research Establishement Ottawa, Ontario, Canada, Technical Report 1280.

[39] Kay, S.M. (1993) Fundamentals of Statistical Signal Processing. Prentice Hall PTR.

[40] Dupuis, D. and Field, C. (1998) Robust Estimation of Extremes. Canadian Journal of Statistics, 26, 199-215. https://doi.org/10.2307/3315505

[41] De Haan, L. and Ferreira, A. (2007) Extreme Value Theory: An Introduction. Springer Science \& Business Media.

[42] Kotz, S. and Nadarajah, S. (2000) Extreme Value Distributions: Theory and Applications. World Scientific. https://doi.org/10.1142/p191 


\section{List of Abbreviations}

CDP: Cyclic Domain Profile

CFD: Cyclostationary Features based Detection

COR: Caractéristique Opérationnelle du Récepteur

CS: Cyclostationnary

ED: Energy based-Detection

FAM: Fast fourier transform Accumulation Method

CF: Crest Factor

FFT: Fast Forier Transform

GEV: Generalized Extreme Value

MLE: Maximum Likewood Estimator

OBRE: Optimal Biased Robust Estimator

OFDM: Strip Spectral Correlation Algorithm

PU: Primary User

ROC: Receiver Operating Characteristic

SNR: Signal Noise Ratio

SU: Secondary User 